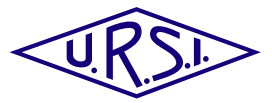

\title{
Wireless Monitoring of a Structural Beam To Be Used for Post-Earthquake Damage Assessment
}

\author{
Burak Ozbey $^{(1)}$, Ozgur Kurc ${ }^{(2)}$, Hilmi Volkan Demir ${ }^{(3)}$, Vakur B. Erturk ${ }^{(3)}$ and Ayhan Altintas*(3) \\ (1) ElectroScience Laboratory, Ohio State University, Columbus, OH 43212 \\ (2) Department of Electrical and Electronics Engineering, Bilkent University, 06800, Ankara, Turkey \\ (3) Department of Civil Engineering, Middle East Technical University, 06800, Ankara, Turkey
}

\begin{abstract}
Wireless monitoring of a standard reinforced concrete beam is shown in a simply supported beam experiment. The passive nested split-ring resonator (NSRR) probes are attached on the reinforcing bars (rebars) within the beam, and an antenna interrogates the probes from outside the beam. The results of the experiment show that the plastic deformation region strain/displacement can be detected by the wireless sensing system. The data collected by the system constitutes an important input for the assessment of the damage that can be observed after earthquakes.
\end{abstract}

\section{Introduction}

The identification of the degree of damage induced in buildings/structures after an earthquake is vital in terms of determining the required repair work or whether an evacuation is necessary or not. Since there does not exist an established quantitative method for understanding the post-earthquake damage, the methods used for this purpose largely rely on observation [1]. In reinforced concrete based buildings, deformations formed on the structural elements (e.g., beams, columns, roof) as well as those formed on the non-structural elements (e.g., furniture, windows, office equipment) are investigated visually. Examining the type and width of a crack or the level of fracture and crushing on concrete, as well as investigating a rupture or a buckling on a reinforcing bar (rebar) inside the concrete (in case it is visible) are examples of the visual methods used by engineers. On the other hand, the effectiveness of visual inspection based methods largely relies on the level of skills and knowledge of the person making the observation, and the differences between the reports prepared by different people of varying degrees of knowledge lead to a difficulties in establishing a standard for understanding the details of the damage [2]. Technological advances in laser scanners have led these devices be used for the purpose of damage assessment in recent years [3, 4]. Although the laser scanners support the observation-based methods with quantitative information, they are far from being an extensively used and feasible option in post-earthquake damage assessment due to their high costs, dimensions and high energy requirements.
In this work, we demonstrate wireless monitoring of a standard reinforced structural beam during a simply supported beam experiment, where the beam undergoes a force up to 25 tons. The vertical force applied to the middle section of the beam standing on two fulcrums at either side causes the whole structure to bend, resulting in tensile strains in the rebars present at the bottom of the beam. These strains, along with the relative displacement, are measured by a wireless sensing system which comprises probes attached on each rebar inside the beam and an external antenna which interrogates these probes. The probes are designed so as to provide a high resolution (being able to detect microstrain-level strain and $\mu \mathrm{m}$-level displacement) and a high sensitivity ( $833 \mathrm{~Hz} /$ Microstrain) by using nested split-ring resonator (NSRR) geometry. Since these strains are proportional with the applied force, and are also closely related with the degree of deformation, an accurate measurement of the strain and the relative displacement between two points is an important tool for identifying the damage that can be experienced after an earthquake.

\section{Wireless Sensing System Architecture}

The wireless sensing system used in this work comprises a transceiver antenna which is connected to a vector network analyzer (VNA), and NSRR probes attached on the rebars within the beam. The system operates based on the principle of near-field electromagnetic coupling between these two elements. By this coupling, a shift in the resonance frequency of the NSRR probe can directly be observed at the antenna response. When the probes are attached on the rebar, an elongation or a contraction of the rebar creates a capacitive change in the NSRR structure, which in turn changes the resonance frequency and can be captured by the antenna. By characterizing this change for different environments (e.g., free space, concrete, rebar grid, etc.), the observed frequency shift can accurately be transformed into displacement, and then to average strain by dividing it to the initial displacement between two attachment points. The near-field electromagnetic coupling makes the high sensitivity and resolution possible in the measurement of displacement and strain [5]. The NSRR probe is a modified version of the regular NSRR geometry first shown in [6]. NSRR architecture makes the system 
completely passive, removing the need for electrical energy. The antenna and the modified NSRR probe employed in this work are shown in Figure 1.

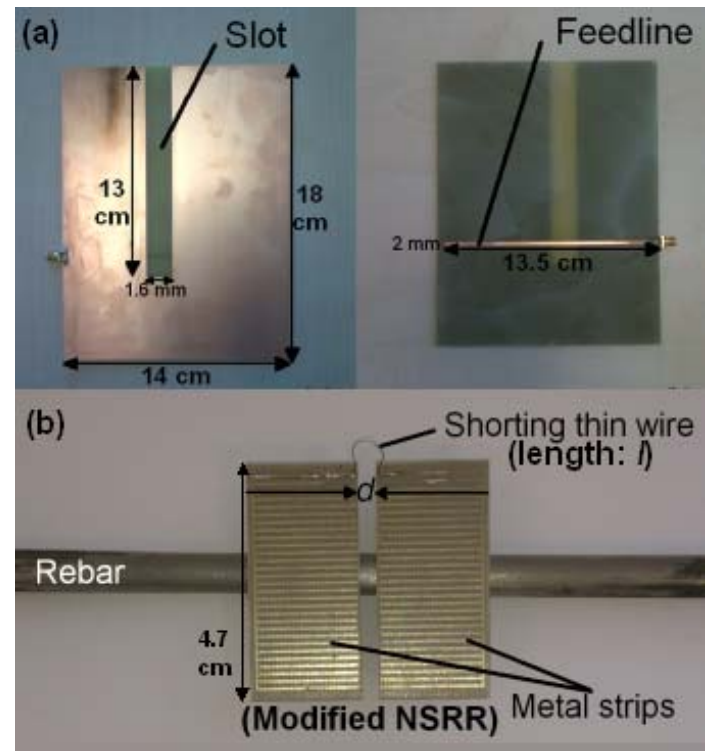

Figure 1. The wireless sensing system components: a) Radiating and feeding sides of the microstrip single-slot antenna, b) Modified nested split-ring resonator (NSRR) probe.

\section{Measurement Setup}

The preparation of the setup for the simply supported beam experiments is shown step by step in Figure 2. First, before the concrete is poured, a cavity is created at the bottom of the beam in order to reach the rebars, onto which the NSRR probes are installed (see Figure 2-a). The direct attachment of the probes on the rebars instead of on the concrete is very critical, since the propagation of strain is hindered through the cracks when concrete is present. On the other hand, since the rebars are elongated or contracted proportionally to the applied force, it is possible to read strain/displacement forming on the beam when the probes are attached on the rebars. At this step, strain gages are also installed at each rebar with wires. Strain gages are wired devices which can operate only at the elastic deformation region at very small strain/displacement levels. They cannot withstand higher levels of loading and come off at the force ranges observed at earthquakes. However, they constitute a means of comparison for this range. As the next step, a protective Plexiglas cover is placed before the rebars (see Figure 2-b). Finally, the beam is enclosed by a plaster layer, and it is loaded by a vertical force at the middle section via a hydraulic piston (see Figure 2c). Finally, the interrogating antenna is brought close to the beam at a fixed position for a good electromagnetic coupling with the probes (see Figure 2-d). It is important that a fixed position is determined for the antenna, so that the disruptive effects of the concrete and the rebar grid do not dominate the coupling signal [7].
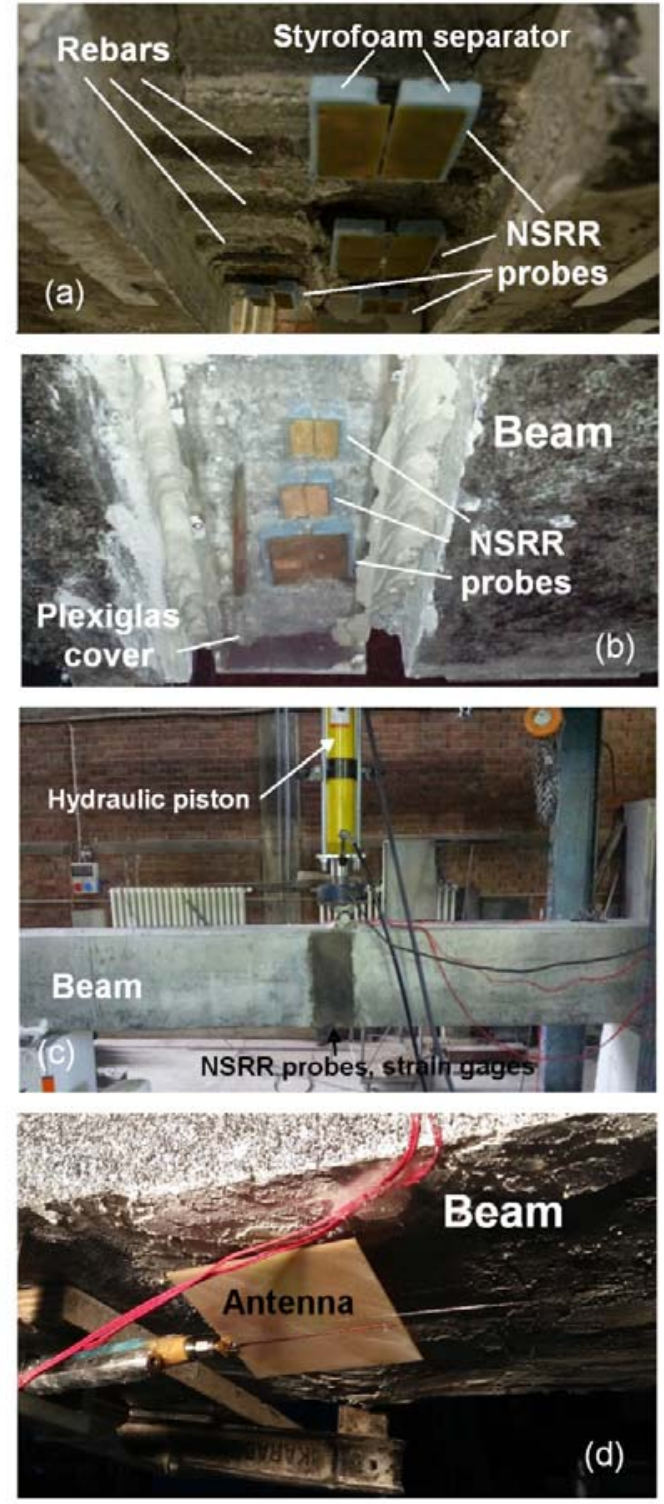

Figure 2. Preparation of the experiment setup: a) Installation of the NSRR probes on the rebars at the bottom of the beam, b) Placement of a protective Plexiglas cover, c) The beam enclosed and placed on two fulcrums at either side, d) Antenna monitors the beam from outside.

\section{Results}

The results of the simply supported beam experiment are shown in Figure 3. First, the beam is loaded with a force slowly increasing up to 25 tons as shown in Figure 3-a. At $t=1125 \mathrm{~s}$, it can clearly be observed in Figure 3-b that the continuous loading causes the rebar to shift from the elastic deformation region to plastic deformation region, initiating irreversible elongation. At this point, it is seen that the strain gages come off and stop producing data. For the whole of the elastic region, the agreement between the sensing system data and the strain gages are observed to be high. After the initiation of the plastic deformation, the loading is increased until $t=2270 \mathrm{~s}$, and it is stopped before 
the beam fails. The strain corresponding to 25 tons of force is 0.023 , and it is the maximum strain observed during the experiment. This corresponds to a relative displacement ( $d$ in Figure 1) of $0.6 \mathrm{~mm}$ on the rebar. The dynamic range of the sensing system is much larger compared to this value [5], which demonstrates that the sensing system can easily record the earthquake-level strains and displacements.
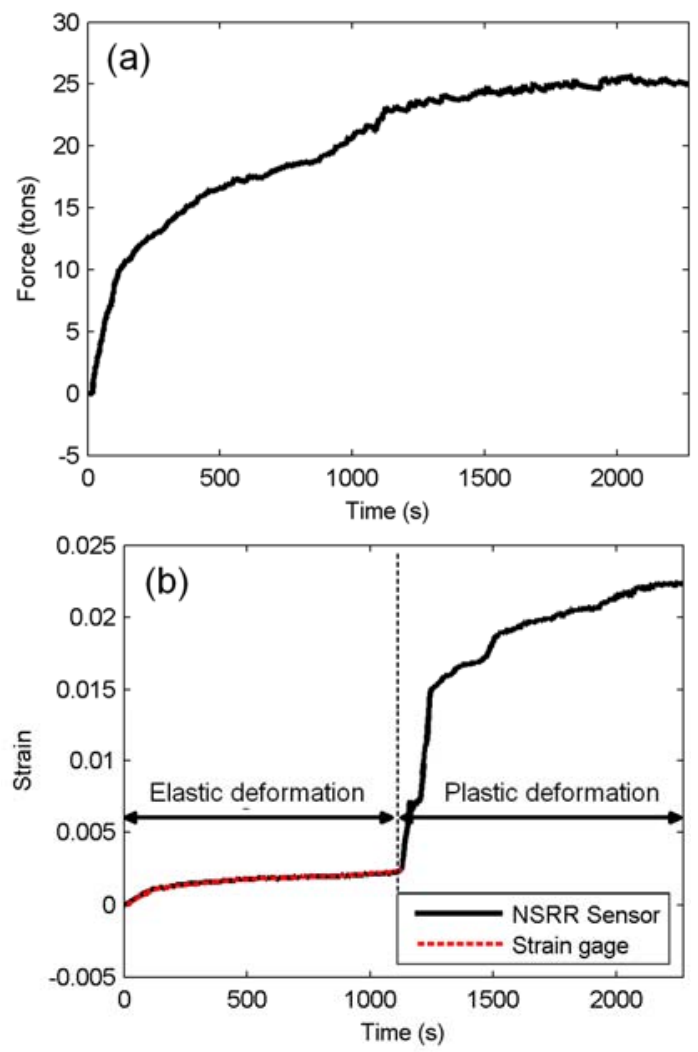

Figure 3. Results of the simply supported beam experiment: a) Applied force versus time, b) The change of strain read from the wireless sensing system compared to strain gages (for elastic region only).

\section{Conclusion}

In this work, we demonstrate wireless measurement of displacement and average strain experienced on the rebars within a reinforced concrete beam during a simply supported beam experiment. The simply supported beam experiment mimics the effect of the earthquake in terms of the force and strain levels that the structural element may undergo. Therefore, it is important that the sensing system can record data during the whole of the experiment without any interruption or malfunction. Currently, there is no established technology to obtain quantitative information regarding the degree of damage after an earthquake. The results of this study show that strain/displacement readings can be acquired through wireless and passive sensing, which may be used in conjunction with current observationbased methods.

\section{Acknowledgements}

This work is partly supported by The Scientific and Technological Research Council of Turkey (TUBITAK) under the EEEAG grant number 112E255.

\section{References}

[1] A. Yakut, A. Erberik, S. Akkar, H. Sucuoglu, and A. Ilki, "Rapid Seismic Assessment Procedures for Turkish Building Stock," SERIES Workshop: "Role of research infrastructures in seismic rehabilitation”, 8-9 February 2012, Istanbul.

[2] B.M. Phares, D.D. Rolander, B.A. Graybeal, and G. Washer, "Reliability of Visual Bridge Inspections," Public Roads, 64, 5, April 2001.

[3] R. Kayen, R. Pack, J. Bay, S. Sugimoto, and H. Tanaka, "Terrestrial-LIDAR Visualization of Surface and Structural Deformations of the 2004 Niigata Ken Chuetsu, Japan, Earthquake,' Earthquake Spectra, 22, S1, pp. 147-162, March 2006, doi: $10.1193 / 1.2173020$

[4] H.S. Park and H.M. Lee, "A New Approach for Health Monitoring of Structures: Terrestrial Laser Scanning,". Computer Aided Civil and Infrastructure Engineering 22, 1, pp. 19-30, January 2007, doi: 10.1111/j.1467-8667.2006.00466.x.

[5] B. Ozbey, E. Unal, H. Ertugrul, O. Kurc, C.M. Puttlitz, V.B. Erturk, A. Altintas, and H.V. Demir, "Wireless Displacement Sensing Enabled by Metamaterial Probes for Remote Structural Health Monitoring,". Sensors 14, 1, pp. 1691-1704, January 2014, doi: 10.3390/s140101691.

[6] R. Melik, E. Unal, N.K. Perkgoz, B. Santoni, D. Kamstock, C. Puttlitz, and H.V. Demir, "Nested Metamaterials for Wireless Strain Sensing,". IEEE Journal of Selected Topics in Quantum Electronics 16, 2, pp. 450-458, March/April 2010, doi: 10.1109/JSTQE.2009.2033391.

[7] B. Ozbey, V.B. Erturk, H.V. Demir, A. Altintas, and O. Kurc, "A Wireless Passive Sensing System for Displacement/Strain Measurement in Reinforced Concrete Members,". Sensors 16, 4, 496, April 2016, doi: 10.3390/s16040496. 